\title{
A DEDICATED INFRARED SYNCHROTRON RADIATION SOURCE AT THE ALS *
}

\author{
W. Barry, A. Biocca, J. M. Byrd ${ }^{\dagger}$ W. Byrne, S. Kwiatkowski, M. C. Martin, W. R. McKinney, H. Nishimura, F. \\ Sannibale, C. Steier, K. Rex, D. Robin, G. Stover, W. Thur, Y. Wu, LBNL, Berkeley, CA 94720, USA \\ Abstract \\ We present ideas for a storage ring dedicated to and \\ optimized for the production of synchrotron radiation over \\ the infrared wavelength range from 1-1000 $\mu \mathrm{m}$. The site \\ for the $66 \mathrm{~m}$ circumference ring is atop the existing ALS \\ booster synchrotron shielding. This area provides enough \\ floor space for both the ring and beamlines, and hutches. \\ We plan to operate the ring in two modes: as a \\ conventional light source and as a superradiant source in \\ coherently over the wavelength range from 200-1000 $\mu \mathrm{m}$. \\ We also hope to minimize the cost of the ring by using \\ existing designs for components such as magnets. \\ The scale of the project is expected to be comparable to \\ an ALS beamline and is considered to be an extension of \\ the capabilities of the ALS facility similar to the \\ superbend project. This paper touches only on the areas of \\ the ring design relevant to IR SR.
} the far-IR. In the conventional mode, our design allows greater transmission of light at longer wavelengths in the far-infrared than typical light sources and significantly improves beam stability. In the superradiant mode, we hope to achieve coherent emission of synchrotron radiation over the $200-1000 \mu \mathrm{m}$ wavelength range by shortening the bunches at very low bunch current

\section{INTRODUCTION}

Infrared spectroscopy and microscopy using synchrotron radiation (SR) has been recognized as a useful analytical technique[1-5] as evidenced by the design and construction of several new beamlines at existing light sources[5]. However, most of these beamlines are being retrofitted to rings originally designed as high brightness soft and hard $\mathrm{x}$-ray sources and thus present several limitations to the performance of the IR beamline. The first is the vertical angular acceptance of the radiation from the beam. Because long wavelength SR has larger vertical opening angle, the vacuum chamber acts as a high pass filter, cutting off much of the long wavelength radiation. This limitation is particularly severe in rings with vacuum chambers with antechambers where most of the far-infrared radiation is suppressed. This is one area in which we hope to make significant gains over the present infrared beamline in the ALS main ring and an area in which there is strong user interest[6-7]. The second limitation is that IR spectromicroscopy using Fourier transform infrared (FTIR) techniques is well known to be sensitive to even small amounts of photon beam motion[8].

This paper describes ideas for the design of a storage ring dedicated to and optimized for the production of infrared SR over the wavelength range from range from 1$1000 \mu \mathrm{m}$. The ring design been inspired by the proliferation of IR beamlines and proposals[9] for producing coherent radiation. The main goals of the design are to increase the range of transmission up to $1 \mathrm{~mm}$ and beyond and to minimize the sources of noise. The

\footnotetext{
*Work supported by the U.S. Department of Energy under Contract Nos. DE-AC03-76SF00098..

$\dagger$ JMByrd@lbl.gov
}

\section{OVERVIEW OF RING DESIGN}

Most synchrotron light sources require a large floor space outside the main ring to accommodate beamlines. However, IR beamlines require relatively little space and prefer to be located as close to the source as possible. Given the layout of the ALS facility as shown in Fig. 1, the ideal location for an IR ring is either in or atop the ALS booster tunnel. Due to clearance restrictions within the tunnel, we have sited the ring on top of the booster shielding. The minimum circumference of a ring that fits on the existing shielding is about $65 \mathrm{~m}$. Full energy injection to the ring can be done from the booster without interfering with injection to the main ring.

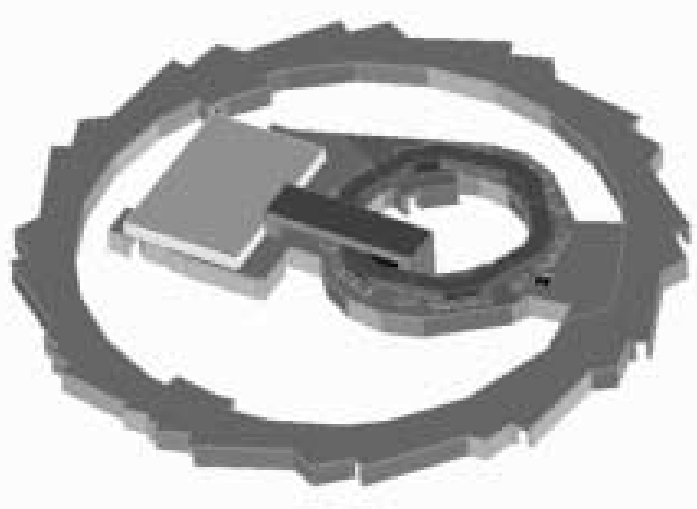

Figure 1: IR ring location atop the ALS booster synchrotron. The ring circumference is $66 \mathrm{~m}$.

Because the brightness and flux over the infrared part of the SR spectrum has little dependence on beam energy, we have chosen $700 \mathrm{MeV}$ as the nominal energy for the conventional mode of ring operation. This choice was determined by trying to find a performance and cost optimization from a variety of effects. Beam stability and lifetime favor higher energies while SR heat loading and accelerator components favor lower energy. 
The lattice is a six-fold symmetric double-bend achromat. General ring parameters are listed in Table 1. Many of the ALS magnets are compatible with the strengths required. One of the significant differences in the lattice compared with third generation machines is that the electron beam emittance can be much larger without degrading the beam brightness over the infrared spectrum. This significantly relaxes the focussing on the lattice which helps in a number of areas such as sensitivity to vibration and power supply jitter, better dynamic aperture, and more robust operation. Furthermore, IR measurement techniques do not require small vertical beam sizes and thus the requirement on the vertical emittance can be strongly relaxed. The larger electron beam emittances strongly reduce Touschek and intrabeam scattering.

\begin{tabular}{cc}
\hline PARAMETER & Value \\
\hline Beam Energy [MeV] & $700(\mathrm{~B} \rho)=2.3367 \mathrm{Tm}$ \\
Current [A] & $<1$ \\
Length [m] & 66 \\
Rev. Freq. [MHz] & 4.54227 \\
RF frequency [MHz] & 49.965 \\
Harmonic Number h & 11 \\
Particles/Bunch (max) & $1.24910^{11}$ \\
Natural Emittance [m rad] & $7.710^{-8}$ \\
Natural Energy Spread & $4.810^{-4}$ \\
$\sigma_{1}$ [mm] & 29.1 \\
$\tau_{\text {gas }}$ [hrs] @ 1 nTorr & 27 \\
Toushek Lifetime [hrs] & $4-5$ \\
Cavity Voltage [kV] & 100 \\
Periodicity & 6 \\
Dipole/quad/sext/period & $2 / 5 / 4$ \\
IR Ports /bend & 3 \\
Bend Angle [deg] & 30 \\
Bend Radius [m] & 1.5578 \\
Tunes (hor/vert) & $4.18 / 2.26$ \\
Synchrotron Freq. [kHz] & 5.5 \\
Natural $\xi$ (hor/vert) & $-5.87 /-6.30$ \\
Momentum Compaction & $7.3510^{-3}$ \\
Damping Time (x/y/z)[ms] & $21.4 / 20.5 / 10.1$ \\
Energy Loss/turn [keV] & 13.65 \\
\hline &
\end{tabular}

Table 1. ALS IR Ring parameters.

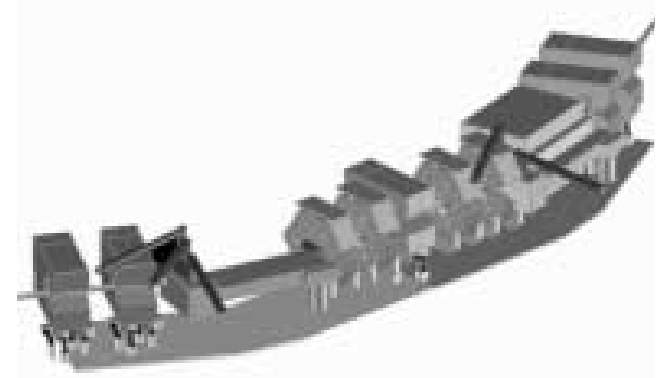

Figure 2: Conceptual view of ring arc sector. IR light ports are extracted from the end of each dipole vacuum chamber.
A view of a ring arc sector is shown in Fig. 2. We expect to use circular stainless pipe for most of the vacuum chamber except for the dipole chambers which are specially designed for maximum vertical and azimuthal acceptance. The ring shielding is made of concrete designed in an inverted U-shape to fit over each sector with a height of about a meter.

\section{BRIGHTNESS AND FLUX}

\subsection{Conventional mode}

Shown in Fig. 3 is the calculated brightness using the ALS IR ring parameters. Also shown is the brightness of the ALS main ring including a $10 \mathrm{mrad}$ vertical aperture. The main improvement in the IR ring comes in the farinfrared. As discussed below, we also expect to increase the horizontal collection angle from $40 \mathrm{mrad}$ to $100 \mathrm{mrad}$.

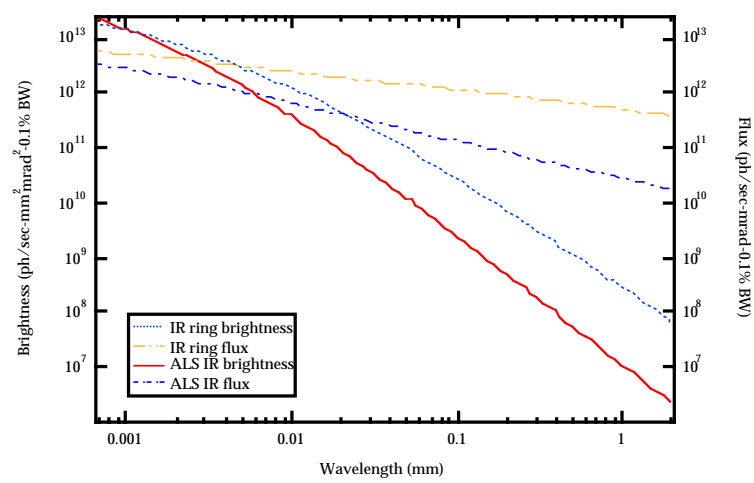

Figure 3. Brightness and flux of the ALS IR ring and existing ALS infrared beamline.

\subsection{Superradiant mode}

When the electron bunch length becomes comparable to the wavelength of emitted SR, the radiation becomes coherent and the power becomes proportional to the square of the number of electrons in the bunch. We refer to the this effect as a superradiant mode of emission.

Many attempts have been made to reach sub-psec bunch lengths in electron rings with little success. However, as Murphy has pointed out, high gains can be made from coherent emission in the far-IR even at very low currents. For example, shown in Fig. 4 is the computed flux in the IR ring for the conventional and superradiant modes with only $1 \mu \mathrm{A}$ of average current in a $150 \mu \mathrm{m}$ bunch. The long wavelength transmission is limited by waveguide cutoff of the vacuum chamber and the short wavelength regime is limited only by limitations in the reduction in bunch length. Note that the flux at $100 \mu \mathrm{m}$ is almost 3 orders of magnitude larger than the IR ring flux at $1 \mathrm{~A}$ at long bunch lengths. The peak current of $40 \mathrm{~A}$ used in this computation has been achieved in many rings. We are actively studying limitations in the bunch length from both the vacuum chamber and radiation impedance.

Although we do not have a specific means of achieving such short bunch lengths, we hope to use a combination of increased RF voltage at higher frequencies and reduction 
in momentum compaction. We believe we have enough flexibility in thelattice and space for additional RF cavities to add ultrashort bunch capability to the ring.

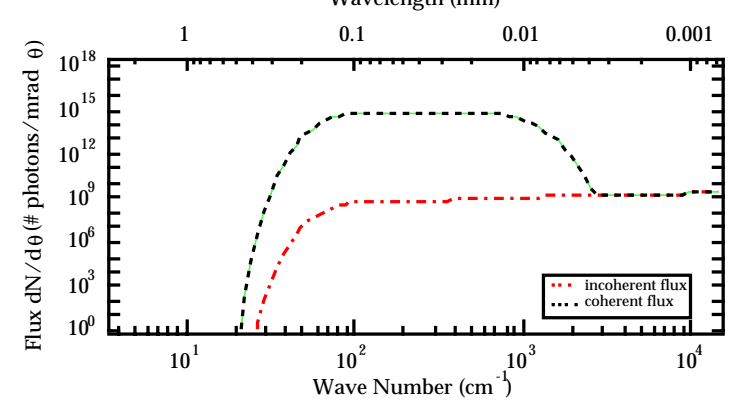

Figure 4. Flux enhancement from coherent emission for a $1 \mu \mathrm{A}$ average bunch current and $150 \mu \mathrm{m}$ bunch length.

\section{BEAMLINE INTEGRATION}

Another of the improvements we hope to achieve with the ALS IR ring is to smoothly integrate the beamline front end with the ring vacuum chamber. This will increase the transmission at long wavelengths and significantly reduce potential sources of beamline motion relative to the electron beam.

Shown in Fig.5 is a drawing of the dipole vacuum chamber, illustrating the extraction of infrared light from the ring. The vertical opening of dipole chamber follows the gradient dipole, resulting in a $100 \mathrm{mrad}$ full vertical aperture, collecting $90 \%$ of the light at $1 \mathrm{~mm}$ wavelength. We plan to use a single first mirror collecting $300 \mathrm{mrad}$ of horizontal angle with the light split into three beamlines by subsequent focussing mirrors. The central cone of hard $\mathrm{X}$-rays will be intercepted by a water-cooled finger to avoid heating the mirror.

Measurements indicate the booster roof shielding is quite stable. Experience with the ALS IR beamline indicates that mounting the beamline and ring on the same stable surface significantly reduces noise. We plan to further reduce noise in ways such as: specifying power supplies according to the more stringent standards of the IR beamline and incorporate feedback on both the optical and electron beams into the initial ring design. We will further reduce any residual photon beam motion by using an optical feedback system currently in use on the ALS IR beamline[11].

\section{SUMMARY AND THANKS}

We are studying ideas for a small ring dedicated and optimized for infrared synchrotron radiation from 1-1000 $\mu \mathrm{m}$ and are currently requesting funding for making a detailed proposal and cost estimate. We would like to thank Jim Murphy for useful discussions of ideas for this

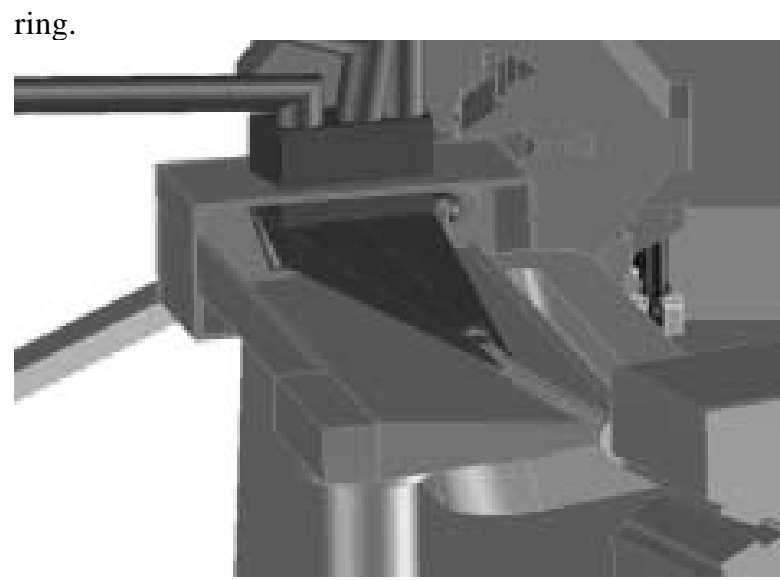

Figure 5. Layout of vacuum chamber with integrated front-end mirror which collects 100v X 300h mrad-mrad.

\section{REFERENCES}

[1] J. A. Reffner, P. A. Martoglio, and G. P. Williams, Rev. Sci. Instrum. 66, 1298 (1995). J.L. Bantignies, G.L. Carr, P. Dumas, L. Miller and G.P. Williams, "Applications of Infrared Microspectroscopy to Geology, Biology and Cosmetics", Synchrotron Radiation News, 11, 31-37 (1998).

[2] G. L. Carr, J. A. Reffner, and G. P. Williams, Rev. Sci. Instrum. 66, 1490 (1995).

[3] W. R. McKinney, C. J. Hirschmugl, H. A. Padmore, T. Lauritzen, N. Andresen, G. Andronaco, R. Patton, and M. Fang, in Accelerator-Based Infrared Sources and Applications (SPIE, San Diego, California, Bellingham, Washington, 1997), Vol. 3153, p. 59.

[4] M. C. Martin and W. R. McKinney, in Applications of Synchrotron Radiation Techniques to Materials Science IV, Materials Research Society, S. M. Mini, S. R. Stock, D. L. Perry, and L. J. Terminello, Eds. (Materials Research Society, San Francisco, California, 1998), Vol. 524, p. 11.

[5] G. P. Williams, Proc. of the 1999 International Society of Optical Engineering, July 1999.

[6] Far-IR at the ALS Workshop Summary, October 1999, LBNL Internal report, LSBL-535.

[7] Future Directions for Far-Infrared Sources Workshop Summary, October 2000, LBNL report, LSBL-573.

[8] J.M. Byrd, M. C. Martin, W.R. McKinney, Proceedings of the 1999 Part. Acc. Conf., New York, p. 495, 1999.

[9] J. Murphy, S. Krinsky, "Millimeter wave coherent synchrotron radiation in an electron storage ring," NIM A 346 (1994) 571-577.

[10] J. M Byrd, M. Chin, M.C. Martin, W. R. McKinney, R. Miller, Proc. of the 1999 International Society of Optical Engineering, July 1999.

[11]"Active Feedback on the Infrared Beamline", ALS 1999 Activity Report pp. 78-79. LBNL-456 